\title{
Les enseignants débutants en adaptation scolaire au Québec: leurs besoins de soutien et le soutien pertinent à offrir
}

\section{Problématique}

L'insertion professionnelle en enseignement correspond à une phase cruciale de la carrière, et les défis inhérents aux premières années dans la profession sont de mieux en mieux connus. Des mesures d'aide à l'insertion apparaissent non seulement comme bénéfiques pour atténuer les difficultés rencontrées, développer des compétences professionnelles, mais aussi pour persévérer en enseignement (Mukamurera et Fontaine, 2017). Au Québec, cependant, les études réalisées à ce jour donnent des résultats ne portant pas sur la situation particulière des enseignantes et enseignants en adaptation scolaire. Puisque ces enseignants travaillent exclusivement avec des élèves en difficulté, leurs besoins de soutien se présentent sans doute plus ou moins différemment que ceux de leurs collègues œuvrant dans les classes dites régulières. Notre question de recherche est la suivante : quels sont les besoins de soutien des enseignants débutants en adaptation scolaire et quel soutien serait-il pertinent de leur offrir? Nos objectifs de recherche sont de décrire et comprendre les besoins de soutien des novices en adaptation scolaire; de décrire l'offre de soutien existante et l'appréciation qu'ils en ont; d'identifier les types de soutien souhaités.

\section{Éléments théoriques}

Nous avons privilégié l'approche multidimensionnelle de l'insertion professionnelle (Mukamurera, Martineau, Bouthiette et Ndoreraho, 2013), laquelle offre une perspective globale de l'insertion impliquant 
les cinq dimensions suivantes: l'intégration en emploi, l'affectation spécifique et les conditions particulières de la tâche, la socialisation organisationnelle, la professionnalité de même que la dimension personnelle et psychologique. Ces dimensions ont été prises en compte dans notre recherche (Giguère, 2018) afin de cerner les besoins de soutien et la nature de l'aide pouvant être offerte aux enseignants débutants en adaptation scolaire. Pour ce qui est du concept de besoin de soutien, il correspond au jugement personnel de l'enseignant quant à la nécessité de recevoir de l'aide par rapport à un aspect de l'insertion ou du travail jugé difficile, négligé, insatisfaisant ou susceptible d'être amélioré.

\section{Méthodologie}

Nous avons réalisé une analyse quantitative des données d'un sous-échantillon de 50 enseignants œuvrant exclusivement en adaptation scolaire et ayant participé à une enquête conduite en 2013 auprès de 250 enseignants débutants issus de divers champs d'enseignement. En complémentarité aux données de l'enquête par questionnaire et pour enrichir la compréhension des résultats quantitatifs, nous avons interviewé cinq enseignantes de notre sous-échantillon, puis réalisé une analyse thématique de leurs propos. Nos questions d'entrevues étaient orientées sur les difficultés vécues en relation avec les cinq dimensions de l'insertion professionnelle et les besoins de soutien qui en découlent, mais nous avons aussi cherché à faire parler les personnes au sujet du soutien reçu et de celui quelles auraient souhaité recevoir.

\section{Résultats et discussion}

Les résultats du questionnaire révèlent que les besoins de soutien ressentis par les novices en adaptation scolaire sont nombreux et variés. Ceux qui arrivent en tête de liste concernent l'évaluation des apprentissages ( $92 \%$ ), le besoin de soutien émotionnel dans les moments difficiles (90\%), le besoin d'être rassuré et confirmé dans ce qu'ils font (90\%), la connaissance des rouages administratifs et syndicaux (90\%) et la gestion des comportements inappropriés des élèves (86 \%). Les propos recueillis lors des entrevues permettent de comprendre leur vécu au quotidien. Les conditions difficiles d'accès aux emplois, les contrats annoncés à la toute dernière minute, le manque de temps pour se préparer, le manque de matériel adapté, l'hétérogénéité des groupes, la prise en charge de programmes très variés et l'instabilité professionnelle sont des irritants exprimés. À cela s'ajoute l'impression d'être dépassé par la charge de travail, la collaboration avec les autres enseignants parfois problématique, mais aussi le sentiment d'être mal préparé par la formation initiale compte tenu de la variété des contextes dans lesquels ils sont appelés à travailler (difficultés des élèves, niveaux, matières et programmes pris en charge, etc.). Cette diversité de contextes de travail semble être à l'origine de plusieurs défis qui constituent également des enjeux en début de carrière chez les enseignants des classes spéciales aux États-Unis (Billingsley, Carlson et Klein, 2004; Youngs, Jones et Low, 2011).

En ce qui concerne l'offre de soutien existante, il en ressort que les mesures proposées dans les milieux sont diversifiées, mais les plus fréquemment offertes (présentation à l'équipe, soutien de la direction, rencontre d'information, trousse d'accueil et observation en classe) ne sont pas nécessairement les plus appréciées. On comprend dès lors l'importance d'offrir des mesures qui tiennent compte des besoins de soutien réels et spécifiques des enseignants (Perry et Hayes, 2011). Pour ce qui est du soutien souhaité, 
il en ressort que l'accès à du matériel adapté est essentiel et qu'un accompagnement individualisé doit être offert systématiquement dès la prise de fonction. L'accessibilité des mesures de soutien, sans égard au statut d'emploi ou au moment de l'embauche (Duchesne et Kane, 2010), tout comme leur diffusion et leur promotion dans les milieux scolaires (Mukamurera et al., 2013) apparaissent comme des conditions à respecter pour encourager l'adhésion et la participation à celles-ci.

Les besoins de soutien des enseignants débutants en adaptation scolaire sont donc de nature plurielle et le soutien à leur offrir devrait tenir compte de l'ensemble de ceux-ci pour favoriser une entrée positive dans la profession.

\section{Références}

Billingsley, B., Carlson, E. et Klein, S. (2004). The working conditions and induction support of early career special educators. Exceptional Children, 7O(3), 333-347. http://dx.doi.org/10.1177/001440290407000305

Duchesne, C. et Kane, R. (2010). Défis de l'insertion professionnelle et dispositifs d'encadrement. McGill Journal of Education, 45(1), 63-80. http://dx.doi.org/10.7202/1000030ar

Giguère, F. (2018). Les enseignantes et enseignants en adaptation scolaire au Québec en situation d'insertion professionnelle : leurs besoins de soutien, le soutien reçu et le soutien soubaité (Mémoire de maîtrise, Université de Sherbrooke). Repéré à https://savoirs.usherbrooke.ca/bitstream/handle/11143/12345/Giguere Fanny MA 2018.pdf?sequence=3

Mukamurera, J. et Fontaine, S. (2017). Les premières années d'enseignement : Réalité professionnelle, besoins de soutien et mesures d'insertion offertes dans les commissions scolaires au Québec. Dans B. Kutsyuruba et K. D. Walker (dir.), The bliss and blisters of early career teaching: a pan-Canadian perspective (p. 181-203). Burlington, ON : Word \& Deed Publishing.

Mukamurera, J., Martineau, S., Bouthiette, M. et Ndoreraho, J. P. (2013). Les programmes d'insertion professionnelle des enseignants dans les commissions scolaires du Québec : portrait et appréciation des acteurs. Éducation et Formation, e-299, 13-35. Repéré à http://revueeducationformation.be/include/download.php?idRevue=17\&idRes=155

Perry, B. et Hayes, K. (2011). The effect of a new teacher induction program on new teachers reported teacher goals for excellence, mobility, and retention rates. International Journal of Educational Leadership Preparation, 6(1), 1-12. Repéré à https://eric.ed.gov/?id=EJ972907

Youngs, P., Jones, N. et Low, M. (2011). How beginning special and general education elementary teachers negotiate role expectations and access professional resources. Teachers College Record, 113(7), 1506-1540.

\section{Pour citer cet article}

Giguère, F. (2019). Les enseignants débutants en adaptation scolaire au Québec : leurs besoins de soutien et le soutien pertinent à offrir. Formation et profession, 27(1),121-123. http://dx.doi.org/10.18162/fp.2019.a163 